\title{
Affective Brain-Computer Interfaces (aBCI 2011)
}

\author{
Christian Mühl ${ }^{1}$, Anton Nijholt ${ }^{1}$, Brendan Allison ${ }^{2}$, Stephen Dunne ${ }^{3}$, and Dirk Heylen ${ }^{1}$ \\ ${ }^{1}$ Human Media Interaction, University of Twente, Enschede, The Netherlands \\ \{muehlc, anijholt, heylen\} @cs. utwente.nl \\ ${ }^{2}$ Technical University, Graz, Austria \\ allisonetugraz.at \\ ${ }^{3}$ Starlab, Barcelona, Spain \\ stephen.dunnedstarlab.es
}

\begin{abstract}
Recently, many groups (see Zander and Kothe. Towards passive brain-computer interfaces: applying brain-computer interface technology to human-machine systems in general. J. Neural Eng., 8, 2011) have worked toward expanding brain-computer interface (BCI) systems to include not only active control, but also passive mental state monitoring to enhance humancomputer interaction (HCI). Many studies have shown that brain imaging technologies can reveal information about the affective and cognitive state of a subject, and that the interaction between humans and machines can be aided by the recognition of those user states. New developments including practical sensors, new machine learning software, and improved interaction with the HCI community are leading us to systems that seamlessly integrate passively recorded information to improve interactions with the outside world.

To achieve robust passive BCIs, efforts from applied and basic sciences have to be combined. On the one hand, applied fields such as affective computing aim to develop applications that adapt to changes in the user states and thereby enrich interaction, leading to a more natural and effective usability. On the other hand, basic research in neuroscience advances our understanding of the neural processes associated with emotions. Similar advancements are made for more cognitive mental states such as attention, workload, or fatigue.

This is the second workshop on affective brain-computer interfaces. The first one was held at ACII 2009 in Amsterdam. Like the first workshop, this one explores the advantages and limitations of using neurophysiological signals as a modality for the automatic recognition of affective and cognitive states, and the possibilities of using this information about the user state in innovative and adaptive applications. Whereas in 2009 the focus was on affective and cognitive state estimation alike, in this 2011 workshop we focus more on the induction, measurement, and use of affective states, i.e. emotions and moods. Hence, the main topics of this workshop are (1) emotion elicitation and data collection for affective BCI, (2) detection of mental state via electroencephalography and other modalities, and (3) adaptive interfaces and affective BCI.

This workshop also seeks to foster interaction among researchers with relevant interests, such as BCI, affective computing, neuro-ergonomics, affective and cognitive neuroscience. These experts present state-of-the-art progress and their visions on the various overlaps between those disciplines.
\end{abstract}

Keywords: affective brain-computer interfacing, passive brain-computer interfacing, affective computing, user state, mental state, cognitive state estimation, emotions, mood, adaptive interfaces, EEG. 